\title{
Doping dependence of the electronic Raman spectra in cuprates
}

\author{
F. Venturini ${ }^{\mathrm{a}}$, M. Opel ${ }^{\mathrm{a}}$, R. Hackl ${ }^{\mathrm{a}}$, H. Berger ${ }^{\mathrm{b}}$, L. Forró $^{\mathrm{b}}$ and B. Revaz ${ }^{\mathrm{c}}$ \\ ${ }^{a}$ Walther Meissner Institute, Bavarian Academy of Sciences, D-85748 Garching, Germany \\ ${ }^{\mathrm{b}}$ EPFL, Ecublens, CH-1015 Lausanne, Switzerland \\ ${ }^{\mathrm{c}} \mathrm{DPMC}$, University of Geneva, CH-1211 Geneva, Switzerland
}

\begin{abstract}
We report electronic Raman scattering measurements on $\mathrm{Bi}_{2} \mathrm{Sr}_{2}\left(\mathrm{Y}_{1-x} \mathrm{Ca}_{x}\right) \mathrm{Cu}_{2} \mathrm{O}_{8+\delta}$ single crystals at different doping levels. The dependence of the spectra on doping and on incoming photon energy is analyzed for different polarization geometries, in the superconducting and in the normal state. We find the scaling behavior of the superconductivity pair-breaking peak with the carrier concentration to be very different in $\mathrm{B}_{1 g}$ and $\mathrm{B}_{2 g}$ geometries. Also, we do not find evidence of any significant variation of the lineshape of the spectra in the overdoped region in both symmetries, while we observe a reduction of the intensity in $\mathrm{B}_{2 g}$ upon decreasing photon energies. The normal state data are analyzed in terms of the memory-function approach. The quasiparticle relaxation rates in the two symmetries display a dependence on energy and temperature which varies with the doping level.
\end{abstract}

\section{Introduction}

Inelastic light-scattering (Raman) experiments reveal a two-particle response of interacting electrons in a similar way as infrared spectroscopy hence combining advantages of angle resolved photoemission spectroscopy (ARPES), by allowing momentum resolution, and of conductivity measurements. Raman studies of the electron properties in cuprates have already been carried out for various doping levels in samples of $\mathrm{Bi}_{2} \mathrm{Sr}_{2}\left(\mathrm{Y}_{1-x} \mathrm{Ca}_{x}\right) \mathrm{Cu}_{2} \mathrm{O}_{8+\delta}$ (Bi-2212), $\mathrm{YBa}_{2} \mathrm{Cu}_{3} \mathrm{O}_{6+x} \quad(\mathrm{Y}-123)$, and $\mathrm{La}_{2-x} \mathrm{Sr}_{x} \mathrm{CuO}_{4}$ (LSCO) 11 1 . However, there are still many unclarified issues as well as considerable improvements in sample quality which motivate a detailed analysis of electronic properties below and above $T_{c}$ with doping $p$. In fact, for the understanding of superconductivity in the cuprates the properties of the normal state are considered to be as crucial as those below $T_{c}$ since the relevant interactions have a characteristic influence on the carriers at all temperatures.

Through the choice of the incident and scattered polarization vectors, Raman scattering is sensitive to different portions of the Fermi surface. By selecting the incident and scattered light polarizations parallel to $x$ and $y$ directions respec- tively, $x$ and $y$ being parallel to the $\mathrm{Cu}-\mathrm{O}$ bonds, the $\mathrm{B}_{2 g}$ symmetry is projected out. In this case the excitations with momenta along the diagonals of the Brillouin zone (BZ) are mainly probed. With polarizations along $x^{\prime} y^{\prime}$ (at $45^{\circ}$ to the $\mathrm{Cu}-\mathrm{O}$ bonds) the $\mathrm{B}_{1 g}$ symmetry is selected. Then the excitation probed are those with momenta along the BZ axes.

In this paper we report electronic Raman scattering measurements in $\mathrm{B}_{1 g}$ and $\mathrm{B}_{2 g}$ polarization geometries at different doping levels in the superconducting and in the normal state. For $T>T_{c}$ the data are analyzed in terms of the memory function approach recently introduced to extract dynamical relaxation rates of the carriers [3].

\section{Experiment}

High quality $\mathrm{Bi}_{2} \mathrm{Sr}_{2}\left(\mathrm{Y}_{1-x} \mathrm{Ca}_{x}\right) \mathrm{Cu}_{2} \mathrm{O}_{8+\delta}$ single crystals were prepared in $\mathrm{ZrO}$ crucibles. They cover a wide doping range as summarized in Table 1. The doping level $p$ is calculated from the relation $T_{c}=T_{c}^{\max }\left[1-82.6(p-0.16)^{2}\right]$ [5].

The experiments were performed in pseudo back-scattering geometry using a standard Raman setup and an $\mathrm{Ar}^{+}$laser for excitation at 458 $\mathrm{nm}, 514 \mathrm{~nm}$ and $528 \mathrm{~nm}$, and $\mathrm{arr}^{+}$laser at 647 $\mathrm{nm}$. To reduce the heating of the sample due to 
Table 1

Bi-2212 single crystals studied.

\begin{tabular}{l|cccccc}
\hline$T_{c}(\mathrm{~K})$ & 57 & 92 & 92 & 78 & 62 & 56 \\
$p$ & 0.09 & 0.15 & 0.16 & 0.20 & 0.22 & 0.23 \\
\hline
\end{tabular}

the absorbed radiation, the power of the laser was maintained below $4 \mathrm{~mW}$. The temperatures given below are corrected for the laser-induced heating as estimated from the ratio of the Stokes to the anti-Stokes intensities. The measured spectra are divided by the Bose-Einstein factor to display the Raman response $\chi^{\prime \prime}$.

\section{Superconducting state}

Fig. 11 shows the $\mathrm{B}_{1 g}$ and $\mathrm{B}_{2 g}$ electronic Raman spectra in differently doped Bi-2212 samples in the superconducting state at approximately 10 $\mathrm{K}$ and in the normal state at approximately $90 \mathrm{~K}$. The data are plotted as a function of energy normalized to the respective transition temperatures of the samples to better visualize the scaling of relevant energies with $T_{c}$.

The superconductivity-induced features in $\mathrm{B}_{1 g}$ and $\mathrm{B}_{2 g}$ symmetries show a very different scaling behavior with the carrier concentration. We will discuss first the $\mathrm{B}_{1 g}$ symmetry results. As the carrier concentration decreases from overdoped, Fig. 1(a), to underdoped, Fig. 1(e), the $\mathrm{B}_{1 g}$ spectrum undergoes substantial modifications. In particular, the intensity is strongly suppressed when reducing the doping level, and for $p<$ 0.15 there is no evidence of superconductivityinduced features (Fig. 1 $1(\mathrm{e})$ ), since there is no observable difference between the superconducting and the normal state spectra. The energy of the superconductivity-induced feature $\omega_{\text {peak }}$, is also strongly affected by doping, going from $\hbar \omega_{\text {peak }} / k_{B} T_{c} \sim 4.5$ in the most overdoped sample (Fig. 1(a)) to $\hbar \omega_{\text {peak }} / k_{B} T_{c} \sim 8.7$ in the slightly underdoped one (Fig. $1(\mathrm{~d})$ ). From these observations we conclude that, in the doping range studied, the peak energy is not proportional to $k_{B} T_{c}$ but decreases monotonically with increasing $p$. It is worth noticing that we observe the same doping dependence in Y-123 samples [6].

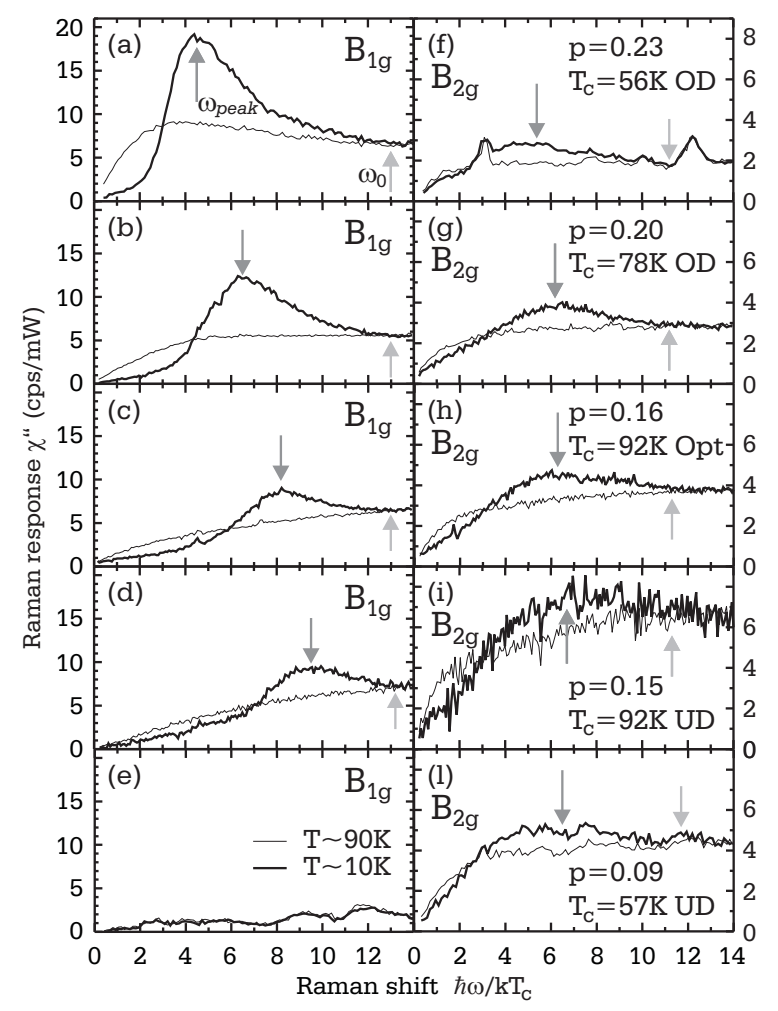

Figure 1. Doping dependence of the Raman spectra in $\mathrm{B}_{1 g}$ and $\mathrm{B}_{2 g}$ symmetries in the superconducting and normal state. The arrows mark the $\omega_{\text {peak }}$ and $\omega_{0}$ energies.

In contrast, the $\mathrm{B}_{2 g}$ spectra show a superconductivity-induced feature at all doping levels as it is evident from Fig. 1](f) to Fig. 11(1). In addition, the maxima of the superconducting spectra $\hbar \omega_{\text {peak }} / k_{B} T_{c}$ are roughly constant between 6 and 6.5 , with the exception of the the most overdoped sample (Fig. 1(f)).

Assuming the superconducting gap to vary as $\Delta(\mathbf{k})=\Delta_{0} / 2\left(\cos \left(k_{x}\right)-\cos \left(k_{y}\right)\right)$, it is possible to extract the maximum $\Delta_{0}$ [7]. In the $\mathrm{B}_{2 g}$ symmetry the measured spectra support $\Delta_{0}$ scaling with $T_{c}$. In addition, the effects of superconductivity are observable up to an energy $\hbar \omega_{0}$ (see Fig. 1), where the superconducting and normal spectra merge, which again scales with $T_{c}$, both in $\mathrm{B}_{1 g}$ 


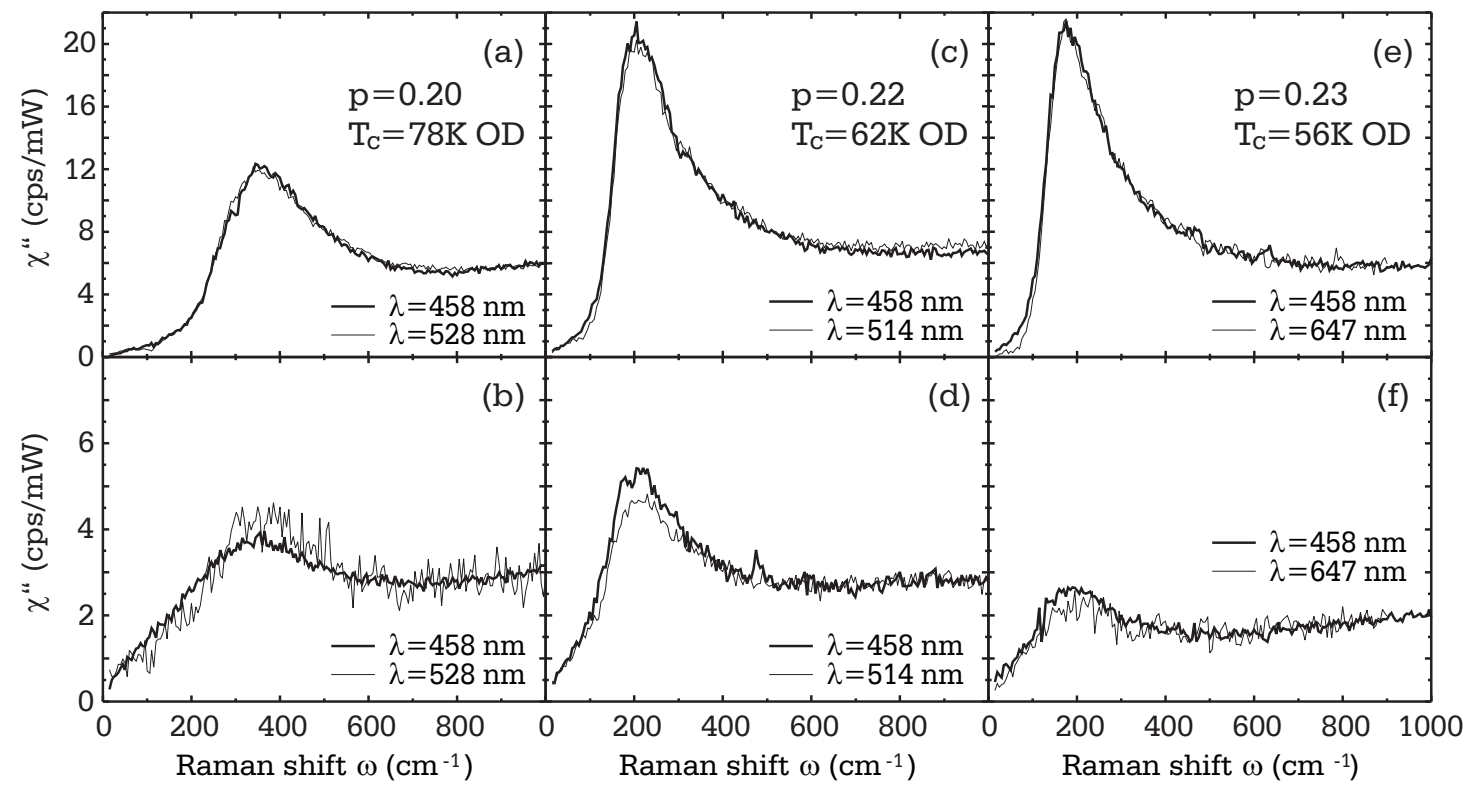

Figure 2. $\mathrm{B}_{1 g}$ and Raman $\mathrm{B}_{1 g}$ spectra of overdoped Bi-2212 samples in the superconducting state $(T \sim 10$ $\mathrm{K})$ at different excitation energies as indicated.

and $\mathrm{B}_{2 g}$ symmetries, at any doping level studied.

The scaling of $\Delta_{0}$ with $T_{c}$ is consistent with the results for the superconducting energy gap derived from the magnetic penetration depth [8], electron tunneling for low bias voltages well below the gap [9], as well as Andreev reflection measurement 10]. However, if the $\mathrm{B}_{1 g}$ peak energy $\hbar \omega_{\text {peak }}$ is considered, Raman scattering seems to reveal an additional energy scale which increases monotonically when decreasing the doping level. This can not be explained in the framework of a simple $d$-wave superconducting energy gap, consistently with the $\mathrm{B}_{2 g}$ symmetry and requires further theoretical work. In addition, the doping dependence of $\omega_{\text {peak }}$ resembles that of the energy scale probed by ARPES and tunneling experiments [11, 12].

It has been suggested that there are two relevant energy scales, namely the single-particle excitation energy, probed by ARPES and tunneling spectroscopies, which increases by decreasing the doping, and a coherence energy scale, obtained for example by Andreev reflections or $\mathrm{B}_{2 g}$ Raman scattering [10]. These two energies approach the same value at high doping levels, where the material is believed to recover a BCS-like behavior, and become increasingly different at low dopings. The interpretation of these energy scales and their relation is still an unsolved question. However, to clearly address these questions more experiments are required to investigate the $\omega_{\text {peak }}$ scaling behavior in the underdoped side of the phase diagram and at which dopings the pair-breaking peak disappears in the $\mathrm{B}_{1 g}$ symmetry.

In order to further investigate the superconductivity-induced features, we have studied the spectra of the three overdoped Bi2212 samples at different excitation energies. In fact, it has been previously argued that resonance properties of the pair-breaking excitations are observable in highly overdoped Bi-2212 samples in $\mathrm{B}_{1 g}$ symmetry, and that they are possibly a signature of an antiferromagnetically correlated Fermi liquid [13].

We analyzed the electronic contribution for excitation energies between $1.6 \mathrm{eV}$ and $2.7 \mathrm{eV}$ in $\mathrm{B}_{1 g}$ 


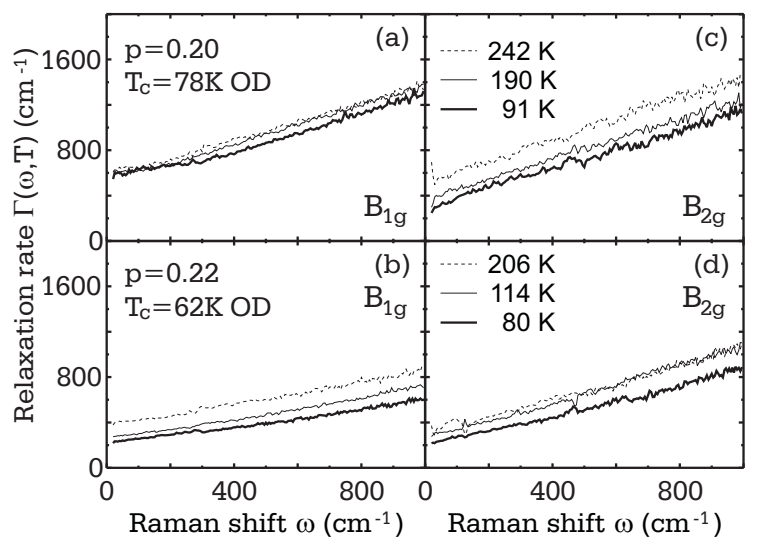

Figure 3. Relaxation rates in $\mathrm{B}_{1 g}((\mathrm{a}),(\mathrm{b}))$ and $\mathrm{B}_{2 g}((\mathrm{c}),(\mathrm{d}))$ symmetries at different temperatures for two overdoped samples.

and $\mathrm{B}_{2 g}$ scattering geometries. Fig. 2 shows the Raman intensity in the $\mathrm{B}_{1 g}$ and $\mathrm{B}_{2 g}$ symmetries for three overdoped Bi-2212 samples. In all cases the overall spectra at wavelengths $\lambda \neq 458 \mathrm{~nm}$ have been multiplied by a factor, ranging from 0.7 to 2 , to adjust the intensity at $800-1000 \mathrm{~cm}^{-1}$ to the spectra at $\lambda=458 \mathrm{~nm}$. In both configurations we do not find any significant variation of the lineshape of the spectra with excitation energy at any doping level. However, we observe a reduction of the overall intensity in the $\mathrm{B}_{2 g}$ symmetry upon decreasing photon energies. Hence, we cannot observe a specific variation of the pair breaking feature with excitation energy neither in Bi-2212 nor in Y-123, except for a small change in the overall cross section.

\section{Normal state}

The Raman continua in the normal state, $T>$ $T_{c}$, (see e.g. Fig. 1) contain information on the dynamical two particle lifetime $\tau$ for different regions in $\mathbf{k}$-space. In Fig. 3 we show the relaxation rates $\Gamma(\omega, T)=1 / \tau$ for two Bi-2212 samples with $p=0.20$ and $p=0.23$, derived as described in [3]. The contributions from the phonons have already been subtracted out.
Both in $B_{1 g}$, Fig. B $3(\mathrm{a}, \mathrm{b})$, and $B_{2 g}$, Fig. 3( symmetries, the variation with frequency of the quasiparticle relaxation rates show only little dependence on momentum and doping. As compared to results at lower doping level [3], there is a tendency to a more quadratic frequency dependence below approximately $400 \mathrm{~cm}^{-1}$ in the $\mathrm{B}_{1 g}$ symmetry possibly indicating more conventional quasiparticle dynamics.

The dependence of the dc limit of the relaxation rates $\Gamma_{0}(T)=\Gamma(\omega \rightarrow 0, T)$ on temperature evolves differently with doping in the two symmetries analyzed. In particular, while $\Gamma_{0}(T)$ decreases with temperature at all doping levels in the $\mathrm{B}_{2 g}$ symmetry, consistently with ordinary and optical transport 14, 15], $\Gamma_{0}^{B_{1 g}}(T)$ is constant or slightly decreasing with $T$ for $p=0.20$ and assumes the $\mathrm{B}_{2 g}$ behavior at $p \geq 0.22$. This disappearance of the anisotropy in the small doping range between 0.20 and 0.22 is directly visible in Fig. 3, where the $p=0.22$ sample shows a strongly suppressed relaxation rate in the $\mathrm{B}_{1 g}$ symmetry (Fig. B(b)). We thus believe that the fundamental change in the carrier dynamics close to $(\pi, 0)$ is an intrinsic property of the electron system itself and is closely related to correlation effects which are found to fade away in this doping range in several other experiments 16 . This implies that the underlying interactions, ordering phenomena, and/or fluctuations must have some structure in momentum space.

\section{Conclusions}

We presented electronic Raman scattering results on $\mathrm{Bi}-2212$. In the $\mathrm{SC}$ state, the $\mathrm{B}_{1 g}$ and $\mathrm{B}_{2 g}$ symmetry spectra are characterized by very different doping dependences of the superconductivity-induced features. While the $\mathrm{B}_{2 g}$ spectra are consistent with a superconducting energy gap with $d$-wave symmetry and a maximum $\Delta_{0}$ scaling with $T_{c}$, the $\mathrm{B}_{1 g}$ symmetry requires further theoretical work. In the normal state the quasiparticles at $(\pi, 0)$ and at $(\pi / 2, \pi / 2)$ are characterized by a qualitatively different doping dependence of the relaxation rates. At high doping levels, $p \geq 0.22$, the anisotropy observed for $p \leq 0.20$ seems to disappear. 
F.V. would like to thank the Gottlieb Daimler and Karl Benz Foundation for financial support.

\section{REFERENCES}

1. C. Kendziora and A. Rosenberg, Phys. Rev. B 52, 9867 (1995).

2. X.K. Chen et al., Phys. Rev. B 56, R513 (1997); J. Naeini et al., Phys. Rev. B 59, 9642 (1999).

3. M. Opel et al., Phys. Rev. B 61, 9752 (2000).

4. S. Sugai and T. Hosokawa, Phys. Rev. Lett. 85, 1112 (2000).

5. J.L. Tallon et al., Phys. Rev. B 51, 12911 (1995).

6. R. Nemetschek et al., Eur. Phys. J. B 5, 495 (1998)

7. T.P. Devereaux and D. Einzel, Phys. Rev. B 51, 16336 (1995).

8. C. Panapogoulos et al., Phys. Rev. B 57, 13422 (1998).

9. C. Renner et al., Phys. Rev. Lett. 80, 149 (1998).

10. G. Deutscher, Nature 397, 410 (1999).

11. N. Miyakawa et al., Phys. Rev. Lett. 80, 157 (1998).

12. J. Mesot et al., Phys. Rev. Lett. 83, 840 (1999).

13. M. Rübhausen et al., Phys. Rev. Lett. 82, 5349 (1999).

14. D.B. Tanner and T. Timusk, in Physical Properties of High-Temperature Superconductors III, ed. by D.M. Ginzberg (World Scientifi, Singapore, 1992)

15. C. Kendziora et al., Phys. Rev. B 48, 3531 (1993).

16. J.L. Tallon ans J.W Loram, Physica C 349, 53 (2001). 